\title{
SYNTHESIS AND PHARMACOLOGICAL TESTING OF SOME NEW DERIVATIVES OF 2,4-(1H,3H)-QUINAZOLINEDIONE (PART II)*
}

\author{
Abdel Ghany A. El-Helby
}

Department of Pharmaceutical Chemistry, Faculty of Pharmacy, Al-Azhar University, Cairo, Egypt

$$
\begin{aligned}
& \text { سبق تشييد عدد كبيز من الإستر ات المختلفة مسن نـواة الكينازولين دايون ودر استها أقربازينيا }
\end{aligned}
$$

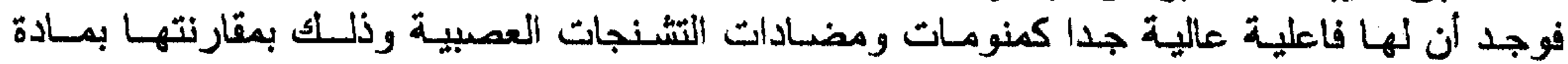

$$
\begin{aligned}
& \text { الفينوباربيتون. }
\end{aligned}
$$

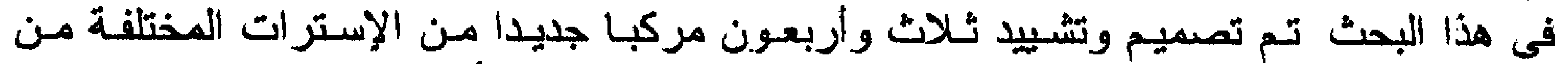

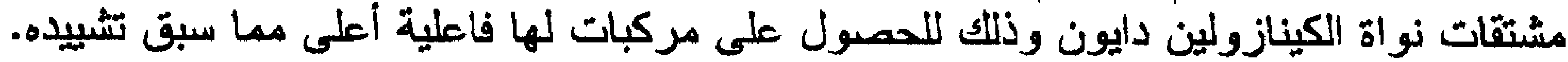

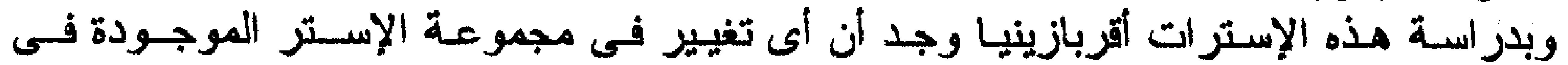

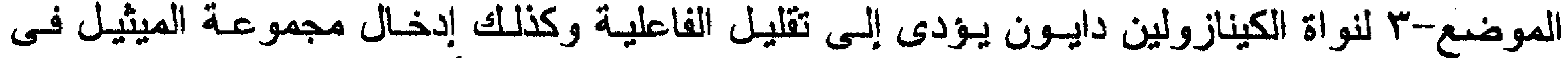

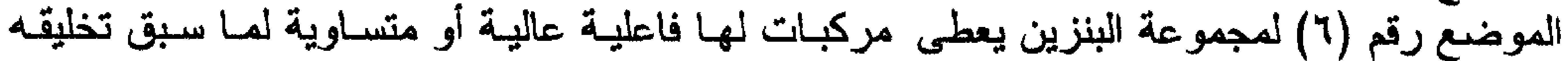

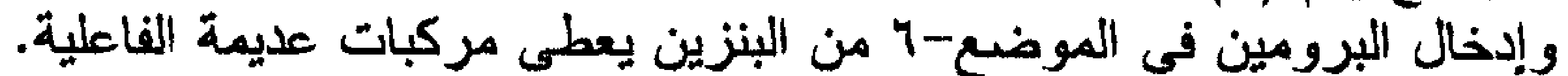

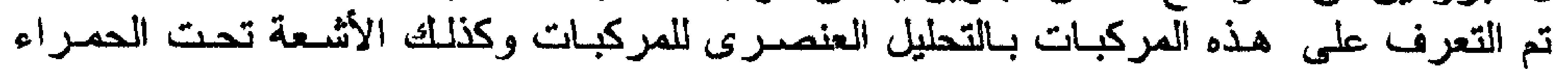

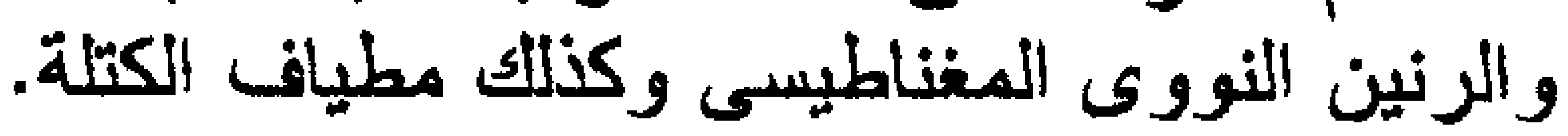

$A$ variety of $2,4(1 \mathrm{H}, 3 \mathrm{H})$ quinazolinediones were converted into the corresponding potassium salts, and then allowed to react with some halogen-containing compounds. The structures of the derivatives thus prepared, were confirmed by elemental, IR, ${ }^{1} H-N M R$ and $M S$ spectral data. Testing for anticonvulsant and hypnotic activities in frogs is also presented.

\section{INTRODUCTION}

Certain derivatives of $2,4(1 \mathrm{H}, 3 \mathrm{H})$ quinazolinediones have been reported to exhibit sedative, tranquilizing, anticonvulsant and hypnotic activities ${ }^{1-7}$. Consequently, it was decided to prepare $2,4(1 \mathrm{H}, 3 \mathrm{H})$ quinazolinediones (III, VII-XI) bearing variable substituents at the 1,3 and 6 positions for possible pharmacological screening in an attempt to study the structure-activity relationship.

\section{EXPERIMENTAL}

Melting points were taken on a Griffen melting points apparatus and are uncorrected. Microanalyses were performed at the Central Laboratory, Faculty of Science, Ain Shams University. IR spectra were recorded on a Buck
Scientific 500 IR Spectrophotometer using $\mathrm{KBr}$ disc. ${ }^{1} \mathrm{H}-\mathrm{NMR}$ spectra were recorded on a Bruker $200 \mathrm{MHz}$ NMR Spectrometer at the Central Laboratory, Faculty of Science, Ain Shams University. MS spectra were recorded at the Microanalytical Center, Faculty of Science, Cairo University, Cairo, Egypt.

According to reported procedures, the following intermediates were prepared: Alkyl chloroacetates $^{8}, \mathrm{~N}$-alkyl ${ }^{9}$ and $\mathrm{N}$-acylanthranilic acids $^{10-12}, \quad 5$-bromo-N-substituted anthranilic acid $^{13-14}, 2,4-(1 \mathrm{H}, 3 \mathrm{H})$-quinazolinedione ${ }^{15}$, 6substituted $2,4-(1 \mathrm{H}, 3 \mathrm{H})$-quinazoline-diones ${ }^{16}$, 1,6-disubstituted $2,4-(1 \mathrm{H}, 3 \mathrm{H})$-quinazolinediones $^{16-20}$, potassium salts of certain 1substituted 2,4-(1H, 3H)-quinazolinediones ${ }^{5,7}$. 1Substituted-3 (2-hydroxyethyl) $2,4(1 \mathrm{H}, 3 \mathrm{H})-$ quinazolinediones $^{21-23}$. 
Potassium salts of 2,4-(1H, $3 \mathrm{H})$ quinazolinediones

General procedure

A solution of the appropriate quinazolinedione (I or V) $(0.01 \mathrm{~mol})$ in absolute ethanol was treated with alcoholic potassium hydroxide solution $(0.02 \mathrm{~mol}$ and $0.01 \mathrm{~mol}$ respectively). The mixture was stirred for 30 minutes at room temperature and left for further 30 minutes. The potassium salts (II) and (VI) were precipitated, filtered, washed several times with absolute ethanol and then dried.

m.p. $\left({ }^{\circ} \mathrm{C}\right):>300$.

Yield: almost quantitative.

1,3-Bis (alkoxycarbonylmethyl)-6-methyl-2,4 (1H, 3H) quinazolinedione (III)(a-e)

A mixture of dipotassium salt (II) $(0.01$ mole) and the suitable alkyl chloroacetes $(0.02$ mole) in DMF $(20 \mathrm{ml})$ was heated on a water bath for two hours. The reaction mixture was cooled, poured into ice-cold water $(200 \mathrm{ml})$, whereupon the crude ester precipitated, filtered and finally recrystallized from ethanol (Table 1).

1-Benzyl and 1-benzoyl-3-(alkoxycarbonylmethyl)-6-bromo-2,4 (1H, 3H) quinazolinediones (VII)(a-j)

A mixture of equimolar quantities of the appropriate potassium salt (VI) (0.01 mole) and the suitable alkyl chloroacetates $(0.01 \mathrm{~mole})$ in DMF $(20 \mathrm{ml})$ was heated on a water bath for two hours. The reaction mixture was cooled, poured into cold water, the precipitated ester was filtered and then crystallized from ethanol (Tabel 1).

\section{1-Alkyl-3-substituted $2,4 \quad(1 \mathrm{H}, 3 \mathrm{H})$-quina- zolinediones (IX)(a-j)}

A mixture of equimolar quantities of 1alky-3-(2-hydroxyethyl)-quinazolinedione (VIII) and the appropriate acyl halide $(0.01 \mathrm{~mole})$ was heated in absolute ethanol $(20 \mathrm{ml})$ for two hours on a water bath. The reaction mixture was cooled, poured into water, filtered and then crystallized from ethanol (Table 1).
Ethyl 4-(1-substituted-quinazolin-2,4-dion-3-yl) crotonate (X) and ethyl 6-(1-substitutedquinazolin-2,4-dion-3-yl) hexanoate (XI)

A mixture of the potassium salts (V) $(0.01$ mole) and ethyl 4-chlorocrotonate ( 0.01 mole) or ethyl 6-chlorohexanoate $(0.01$ mole) was heated in DMF $(20 \mathrm{ml})$ on a water bath for 3 hours. The reaction mixture was then cooled, poured into cold water. The solid produced was filtered then crystallized from ethanol to afford the new target esters (X) or (XI) respectively (Table 1).

For the preparation of these new compounds, scheme (I) was adopted.

Structures of all new derivatives of 2,4 $(1 \mathrm{H}, 3 \mathrm{H}$ )-quinazolinedione (III, VII-XI) were substantiated from elemental (Table 1) and spectral data (Table 2).

\section{RESULTS AND DISCUSSION}

5-Methylanthranilic acid was converted into 6-methyl-2,4- $(1 \mathrm{H}, 3 \mathrm{H})$ quinazolinedione $(\mathrm{I})$, the potassium salt (II) of which was allowed to react with alkyl chloroacetates to obtain the final compounds (III). Other pathways for the synthesis of 1,3,6-trisubstituted 2,4-(1H, 3H) quinazolinediones were followed starting with 5substituted anthranilic acid, which was initially $\mathrm{N}$-alkylated for $\mathrm{N}$-acylated, then reacted with urea to produce the 1,6-disubstituted 2,4 $(1 \mathrm{H}$, $3 \mathrm{H})$ quinazolinediones $(\mathrm{V})$, the latter potassium salts (VI) reacted with alkyl chloroacetates to afford VII. Elongation of the side chain at the 3position of the $2,4(1 \mathrm{H}, 3 \mathrm{H})$ quinazolinedione ring system was attempted by allowing (VI) to react with B-chloroethanol to get the Bhydroxyethyl derivatives (VIII), which were finally esterified with acyl halides into the target products (IX). Furthermore, in the same broad line, the potassium salts (VI) were allowed to react with ethyl chlorocrotonate and ethyl $\omega$ chlorohexanoate whereupon the respective esters (X and $\mathrm{XI}$ ) were obtained in good yields.

\section{Pharmacological testing \\ 1- Hypnotic activity \\ Hypnotic activity of the new compounds was primarily determined in frogs by the}


Scheme I

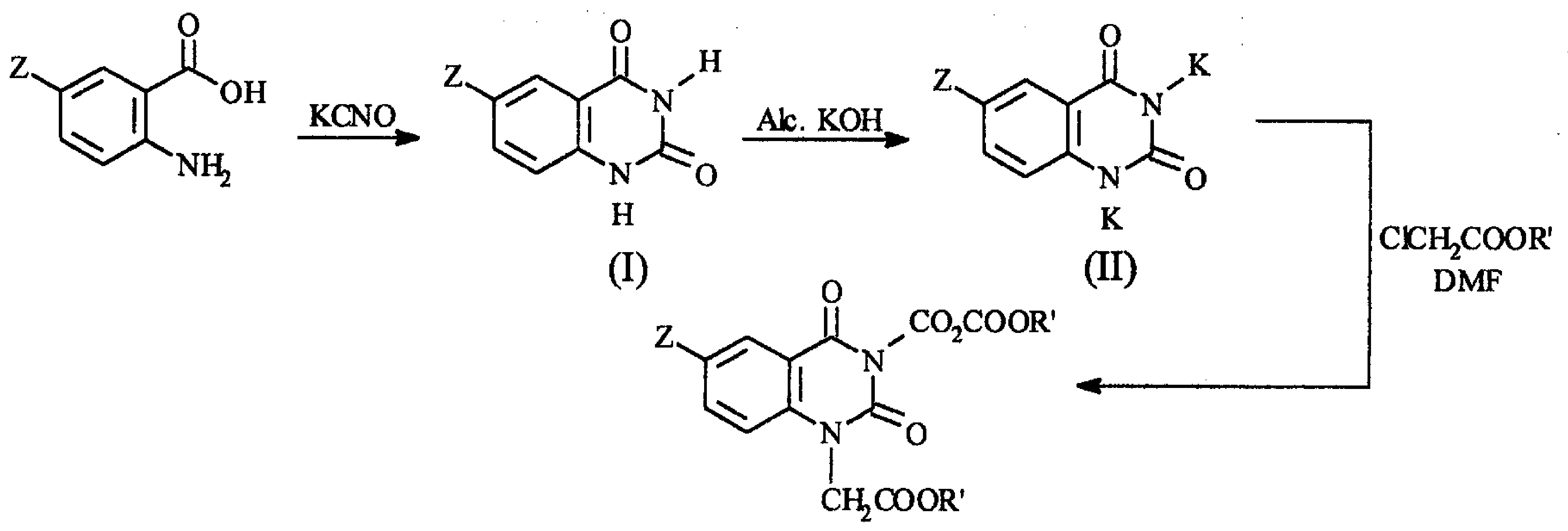

(III)(a-e)

$\overbrace{\mathrm{NH}_{2}}^{\stackrel{\mathrm{N}}{\mathrm{R}-\mathrm{X}}}$<smiles>[R]Nc1ccc([Z])cc1C(=O)O</smiles>

(IV)<smiles></smiles>

(V)

Alc. $\mathrm{KOH}$<smiles>[R]OC(=O)Cn1c(=O)[nH]c2ccc([Z])cc2c1=O</smiles>

(VII)(a-j)<smiles>CCCCCC(=O)O[Na]</smiles>
DMF<smiles>[R]c1[nH]c(=O)n([R])c2ccc([Z])c(=O)c1-2</smiles>

(VI)

$\mathrm{ClCH}_{2} \mathrm{CH}_{2} \mathrm{OH}$<smiles>[R2]OOCCn1c(=O)[nH]c2ccc([Z])cc2c1=O</smiles>

$(I X)(a-j)$

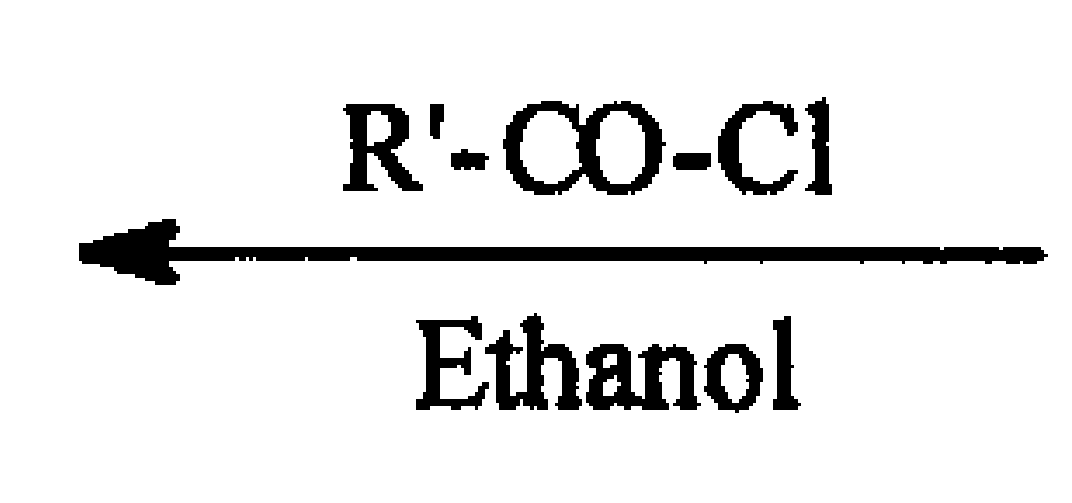

thanol<smiles>[R]c1ccc2[nH]c(=O)n(CCO)c(=O)c2c1</smiles>

(VIII)<smiles>[R]c1ccc2[nH]c(=O)n(CC(=O)OCC)c(=O)c2c1</smiles>

$(\mathrm{XI})(\mathrm{a}-\mathrm{i})$ 
Table 1: $\quad$ 1,3,6-trisubstituted $2,4(1 \mathrm{H}, 3 \mathrm{H})$ quinazolinedione derivatives.

\begin{tabular}{|c|c|c|c|c|c|c|c|c|c|c|}
\hline \multirow{2}{*}{$\begin{array}{c}\text { Comp. } \\
\text { No. }\end{array}$} & \multirow{2}{*}{$\mathrm{Z}$} & \multirow{2}{*}{$\mathbf{R}$} & \multirow{2}{*}{$\mathbf{R}^{\prime}$} & \multirow{2}{*}{$\underset{ }{\text { M.P }}{ }^{\circ} \mathrm{C}$} & \multirow{2}{*}{$\begin{array}{c}\text { Yield } \\
\%\end{array}$} & \multirow{2}{*}{$\begin{array}{l}\text { Molecular } \\
\text { formula }\end{array}$} & \multirow{2}{*}{ M.wt. } & \multicolumn{3}{|c|}{$\begin{array}{c}\text { Analysis } \\
\text { Calcd./found } \\
\end{array}$} \\
\hline & & & & & & & & $\mathrm{C} \%$ & $\mathrm{H} \%$ & N\% \\
\hline III-a & $\mathrm{CH}_{3}$ & - & $\mathrm{CH}_{3}$ & $90-1$ & 76 & $\mathrm{C}_{15} \mathrm{H}_{16} \mathrm{~N}_{2} \mathrm{O}_{6}$ & 320 & $\begin{array}{l}56.25 \\
56.30 \\
\end{array}$ & $\begin{array}{l}5.00 \\
5.10\end{array}$ & $\begin{array}{l}8.75 \\
8.60\end{array}$ \\
\hline b & $\mathrm{CH}_{3}$ & - & $\mathrm{C}_{2} \mathrm{H}_{5}$ & $88-9$ & 77 & $\mathrm{C}_{17} \mathrm{H}_{20} \mathrm{~N}_{2} \mathrm{O}_{6}$ & 348 & $\begin{array}{l}58.62 \\
58.60\end{array}$ & $\begin{array}{l}5.74 \\
5.60\end{array}$ & $\begin{array}{l}8.04 \\
8.10\end{array}$ \\
\hline c & $\mathrm{CH}_{3}$ & - & $\mathrm{C}_{3} \mathrm{H}_{7}(\mathrm{n})$ & $70-1$ & 45 & $\mathrm{C}_{19} \mathrm{H}_{24} \mathrm{~N}_{2} \mathrm{O}_{6}$ & 376 & $\begin{array}{l}60.63 \\
60.60\end{array}$ & $\begin{array}{l}6.38 \\
6.40\end{array}$ & $\begin{array}{l}7.44 \\
7.40\end{array}$ \\
\hline d & $\mathrm{CH}_{3}$ & -. & $\mathrm{C}_{3} \mathrm{H}_{7}$ (iso) & 105 & 72 & $\mathrm{C}_{19} \mathrm{H}_{24} \mathrm{~N}_{2} \mathrm{O}_{6}$ & 376 & $\begin{array}{l}60.63 \\
60.50\end{array}$ & $\begin{array}{l}6.38 \\
6.30\end{array}$ & $\begin{array}{l}7.44 \\
7.60\end{array}$ \\
\hline e & $\mathrm{CH}_{3}$ & - & $\mathrm{C}_{4} \mathrm{H}_{9}(\mathrm{n})$ & $68-9$ & 62 & $\mathrm{C}_{21} \mathrm{H}_{28} \mathrm{~N}_{2} \mathrm{O}_{6}$ & 404 & $\begin{array}{l}62.37 \\
62.20\end{array}$ & $\begin{array}{l}6.93 \\
6.80\end{array}$ & $\begin{array}{l}6.93 \\
7.00\end{array}$ \\
\hline VII-a & $\mathrm{Br}$ & $\mathrm{C}_{6} \mathrm{H}_{5} \mathrm{CH}_{2}$ & $\mathrm{CH}_{3}$ & 110 & 42 & $\mathrm{C}_{18} \mathrm{H}_{15} \mathrm{BrN}_{2} \mathrm{O}_{4}$ & 403 & $\begin{array}{l}53.59 \\
53.70\end{array}$ & $\begin{array}{l}3.72 \\
3.80\end{array}$ & $\begin{array}{l}6.94 \\
6.90\end{array}$ \\
\hline b & $\mathrm{Br}$ & $\mathrm{C}_{6} \mathrm{H}_{5} \mathrm{CH}_{2}$ & $\mathrm{C}_{2} \mathrm{H}_{5}$ & $125-6$ & 44 & $\mathrm{C}_{19} \mathrm{H}_{17} \mathrm{BrN}_{2} \mathrm{O}_{4}$ & 417 & $\begin{array}{l}54.67 \\
54.70\end{array}$ & $\begin{array}{l}4.07 \\
4.00\end{array}$ & $\begin{array}{l}6.71 \\
6.90\end{array}$ \\
\hline c & $\mathrm{Br}$ & $\mathrm{C}_{6} \mathrm{H}_{5} \mathrm{CH}_{2}$ & $\mathrm{C}_{3} \mathrm{H}_{7}(\mathrm{n})$ & 112 & 45 & $\mathrm{C}_{20} \mathrm{H}_{19} \mathrm{BrN}_{2} \mathrm{O}_{4}$ & 431 & $\begin{array}{l}55.68 \\
56.00\end{array}$ & $\begin{array}{l}4.40 \\
3.80\end{array}$ & $\begin{array}{l}6.49 \\
6.40\end{array}$ \\
\hline d & $\mathrm{Br}$ & $\mathrm{C}_{6} \mathrm{H}_{5} \mathrm{CH}_{2}$ & $\mathrm{C}_{3} \mathrm{H}_{7}$ (iso) & $143-4$ & 67 & $\mathrm{C}_{20} \mathrm{H}_{19} \mathrm{BrN}_{2} \mathrm{O}_{4}$ & 431 & $\begin{array}{l}55.68 \\
55.70\end{array}$ & $\begin{array}{l}4.40 \\
4.00\end{array}$ & $\begin{array}{l}6.49 \\
6.70\end{array}$ \\
\hline e & $\mathrm{Br}$ & $\mathrm{C}_{6} \mathrm{H}_{5} \mathrm{CH}_{2}$ & $\mathrm{C}_{4} \mathrm{H}_{9}(\mathrm{n})$ & 75 & 52 & $\mathrm{C}_{21} \mathrm{H}_{21} \mathrm{BrN}_{2} \mathrm{O}_{4}$ & 445 & $\begin{array}{l}56.62 \\
57.00\end{array}$ & $\begin{array}{l}4.71 \\
4.40\end{array}$ & $\begin{array}{l}6.29 \\
6.30\end{array}$ \\
\hline f & $\mathrm{Br}$ & $\mathrm{C}_{6} \mathrm{H}_{5} \mathrm{CO}$ & $\mathrm{CH}_{3}$ & 130 & 53 & $\mathrm{C}_{18} \mathrm{H}_{13} \mathrm{BrN}_{2} \mathrm{O}_{5}$ & 417 & $\begin{array}{l}51.79 \\
51.80\end{array}$ & $\begin{array}{l}3.11 \\
3.20\end{array}$ & $\begin{array}{l}6.71 \\
6.70\end{array}$ \\
\hline $\mathrm{g}$ & $\mathrm{Br}$ & $\mathrm{C}_{6} \mathrm{H}_{5} \mathrm{CO}$ & $\mathrm{C}_{2} \mathrm{H}_{5}$ & $112-3$ & 65 & $\mathrm{C}_{19} \mathrm{H}_{15} \mathrm{BrN}_{2} \mathrm{O}_{5}$ & 431 & $\begin{array}{l}52.90 \\
53.00\end{array}$ & $\begin{array}{l}3.48 \\
3.30\end{array}$ & $\begin{array}{l}6.49 \\
6.50\end{array}$ \\
\hline $\mathbf{h}$ & $\mathrm{Br}$ & $\mathrm{C}_{6} \mathrm{H}_{5} \mathrm{CO}$ & $\mathrm{C}_{3} \mathrm{H}_{7}(\mathrm{n})$ & $115-6$ & 45 & $\mathrm{C}_{20} \mathrm{H}_{17} \mathrm{BrN}_{2} \mathrm{O}_{5}$ & 445 & $\begin{array}{l}53.93 \\
54.00\end{array}$ & $\begin{array}{l}3.82 \\
3.80 \\
\end{array}$ & $\begin{array}{l}6.29 \\
6.50\end{array}$ \\
\hline $\mathrm{i}$ & $\mathrm{Br}$ & $\mathrm{C}_{6} \mathrm{H}_{5} \mathrm{CO}$ & $\mathrm{C}_{3} \mathrm{H}_{7}$ (iso) & $125-6$ & 55 & $\mathrm{C}_{20} \mathrm{H}_{17} \mathrm{BrN}_{2} \mathrm{O}_{5}$ & 445 & $\begin{array}{l}53.93 \\
53.60 \\
\end{array}$ & $\begin{array}{l}3.82 \\
3.50 \\
\end{array}$ & $\begin{array}{l}6.29 \\
6.00\end{array}$ \\
\hline $\mathrm{j}$ & $\mathrm{Br}$ & $\mathrm{C}_{6} \mathrm{H}_{5} \mathrm{CO}$ & $\mathrm{C}_{4} \mathrm{H}_{9}(\mathrm{n})$ & 95 & 85 & $\mathrm{C}_{21} \mathrm{H}_{19} \mathrm{BrN}_{2} \mathrm{O}_{5}$ & 459 & $\begin{array}{l}54.90 \\
55.00\end{array}$ & $\begin{array}{l}4.13 \\
4.00\end{array}$ & $\begin{array}{l}6.10 \\
6.30\end{array}$ \\
\hline IX-a & $\mathbf{H}$ & $\mathrm{C}_{2} \mathrm{H}_{5}$ & $\mathrm{CH}_{3}$ & 97 & 70 & $\mathrm{C}_{14} \mathrm{H}_{16} \mathrm{~N}_{2} \mathrm{O}_{4}$ & 276 & $\begin{array}{l}60.86 \\
60.76\end{array}$ & $\begin{array}{l}5.79 \\
5.80\end{array}$ & $\begin{array}{l}10.14 \\
10.00\end{array}$ \\
\hline b & $\mathbf{H}$ & $\mathrm{C}_{6} \mathrm{H}_{5}$ & $\mathrm{C}_{2} \mathrm{H}_{5}$ & 100 & 80 & $\mathrm{C}_{19} \mathrm{H}_{18} \mathrm{~N}_{2} \mathrm{O}_{4}$ & 338 & $\begin{array}{l}67.45 \\
67.80 \\
\end{array}$ & $\begin{array}{l}5.32 \\
5.30 \\
\end{array}$ & $\begin{array}{l}8.28 \\
8.30\end{array}$ \\
\hline c & $\mathrm{H}$ & $\mathrm{C}_{6} \mathrm{H}_{5}$ & $\mathrm{C}_{2} \mathrm{H}_{5} \mathrm{CH}_{2}$ & $75-6$ & 77 & $\mathrm{C}_{20} \mathrm{H}_{20} \mathrm{~N}_{2} \mathrm{O}_{4}$ & 352 & $\begin{array}{l}68.18 \\
68.30\end{array}$ & $\begin{array}{l}5.68 \\
5.50\end{array}$ & $\begin{array}{l}7.95 \\
7.60\end{array}$ \\
\hline
\end{tabular}


Table 1: Continued

\begin{tabular}{|c|c|c|c|c|c|c|c|c|c|c|}
\hline \multirow{2}{*}{$\begin{array}{l}\text { Comp. } \\
\text { No. }\end{array}$} & \multirow[t]{2}{*}{$\mathrm{Z}$} & \multirow[t]{2}{*}{$\mathbf{R}$} & \multirow[t]{2}{*}{$\mathbf{R}^{\prime}$} & \multirow{2}{*}{$\begin{array}{l}\text { M.P } \\
{ }^{\circ} \mathrm{C}\end{array}$} & \multirow{2}{*}{$\begin{array}{c}\text { Yield } \\
\%\end{array}$} & \multirow{2}{*}{$\begin{array}{l}\text { Molecular } \\
\text { formula }\end{array}$} & \multirow[t]{2}{*}{ M.wt. } & \multicolumn{3}{|c|}{$\begin{array}{c}\text { Analysis } \\
\text { Calcd./found }\end{array}$} \\
\hline & & & & & & & & $\mathrm{C} \%$ & $\mathrm{H} \%$ & N\% \\
\hline d & $\mathrm{H}$ & $\mathrm{C}_{2} \mathrm{H}_{5}$ & $\mathrm{C}_{6} \mathrm{H}_{4}-\mathrm{Cl}(\mathrm{p})$ & 135 & 90 & $\mathrm{C}_{19} \mathrm{H}_{17} \mathrm{ClN}_{2} \mathrm{O}_{4}$ & 372.5 & $\begin{array}{l}61.20 \\
61.30 \\
\end{array}$ & $\begin{array}{l}4.56 \\
4.50 \\
\end{array}$ & $\begin{array}{l}7.51 \\
7.30 \\
\end{array}$ \\
\hline e & $\mathbf{H}$ & $\mathrm{C}_{2} \mathrm{H}_{5}$ & $\mathrm{C}_{6} \mathrm{H}_{4}-\mathrm{NO}_{2}(\mathrm{p})$ & 220 & 85 & $\mathrm{C}_{19} \mathrm{H}_{17} \mathrm{~N}_{3} \mathrm{O}_{6}$ & 383 & $\begin{array}{l}59.53 \\
59.60 \\
\end{array}$ & $\begin{array}{l}4.43 \\
4.50 \\
\end{array}$ & $\begin{array}{l}10.90 \\
11.00\end{array}$ \\
\hline f & $\mathbf{H}$ & $\mathrm{CH}_{2} \mathrm{CH}=\mathrm{CH}_{2}$ & $\mathrm{CH}_{3}$ & $70-1$ & 82 & $\mathrm{C}_{15} \mathrm{H}_{16} \mathrm{~N}_{2} \mathrm{O}_{4}$ & 288 & $\begin{array}{l}62.50 \\
62.50 \\
\end{array}$ & $\begin{array}{l}5.55 \\
5.30 \\
\end{array}$ & $\begin{array}{r}9.72 \\
10.00 \\
\end{array}$ \\
\hline $\mathrm{g}$ & $\mathbf{H}$ & $\mathrm{CH}_{2} \mathrm{CH}=\mathrm{CH}_{2}$ & $\mathrm{C}_{6} \mathrm{H}_{5}$ & 110 & 55 & $\mathrm{C}_{20} \mathrm{H}_{18} \mathrm{~N}_{2} \mathrm{O}_{4}$ & 350 & $\begin{array}{l}68.57 \\
68.70 \\
\end{array}$ & $\begin{array}{l}5.14 \\
5.00\end{array}$ & $\begin{array}{l}8.00 \\
7.90\end{array}$ \\
\hline $\mathrm{h}$ & $\mathrm{H}$ & $\mathrm{CH}_{2} \mathrm{CH}=\mathrm{CH}_{2}$ & $\mathrm{C}_{6} \mathrm{H}_{5} \mathrm{CH}_{2}$ & $90-1$ & 45 & $\mathrm{C}_{21} \mathrm{H}_{20} \mathrm{~N}_{2} \mathrm{O}_{4}$ & 364 & $\begin{array}{l}69.23 \\
69.00 \\
\end{array}$ & $\begin{array}{l}5.49 \\
5.50\end{array}$ & $\begin{array}{l}7.69 \\
8.00\end{array}$ \\
\hline $\mathrm{i}$ & $\mathrm{H}$ & $\mathrm{CH}_{2} \mathrm{CH}=\mathrm{CH}_{2}$ & $\mathrm{C}_{6} \mathrm{H}_{4}-\mathrm{Cl}(\mathrm{p})$ & 175 & 76 & $\mathrm{C}_{20} \mathrm{H}_{17} \mathrm{ClN}_{2} \mathrm{O}_{4}$ & 384.5 & $\begin{array}{l}62.41 \\
62.25\end{array}$ & $\begin{array}{l}4.42 \\
4.42\end{array}$ & $\begin{array}{l}7.28 \\
7.00\end{array}$ \\
\hline $\mathrm{j}$ & $\mathrm{H}$ & $\mathrm{CH}_{2} \mathrm{CH}=\mathrm{CH}_{2}$ & $\mathrm{C}_{6} \mathrm{H}_{4}-\mathrm{NO}_{2}(\mathrm{p})$ & 200 & 50 & $\mathrm{C}_{20} \mathrm{H}_{17} \mathrm{~N}_{3} \mathrm{O}_{6}$ & 395 & $\begin{array}{l}60.75 \\
61.00 \\
\end{array}$ & $\begin{array}{r}4.30 \\
4.00 \\
\end{array}$ & $\begin{array}{l}10.63 \\
10.60 \\
\end{array}$ \\
\hline$X-a$ & $\mathbf{H}$ & $\mathrm{CH}_{3}$ & - & 50 & 76 & $\mathrm{C}_{15} \mathrm{H}_{16} \mathrm{~N}_{2} \mathrm{O}_{4}$ & 288 & $\begin{array}{l}62.50 \\
62.50 \\
\end{array}$ & $\begin{array}{l}5.55 \\
5.50 \\
\end{array}$ & $\begin{array}{l}9.72 \\
9.50 \\
\end{array}$ \\
\hline b & $\mathbf{H}$ & $\mathrm{C}_{2} \mathrm{H}_{5}$ & - & $80-1$ & 69 & $\mathrm{C}_{16} \mathrm{H}_{18} \mathrm{~N}_{2} \mathrm{O}_{4}$ & 302 & $\begin{array}{l}63.57 \\
63.40\end{array}$ & $\begin{array}{l}5.96 \\
6.00\end{array}$ & $\begin{array}{l}9.27 \\
9.00\end{array}$ \\
\hline c & $\mathrm{H}$ & $\mathrm{C}_{3} \mathrm{H}_{7}(\mathrm{n})$ & - & $61-1$ & 75 & $\mathrm{C}_{17} \mathrm{H}_{20} \mathrm{~N}_{2} \mathrm{O}_{4}$ & 316 & $\begin{array}{l}64.55 \\
64.50 \\
\end{array}$ & $\begin{array}{l}6.32 \\
6.30 \\
\end{array}$ & $\begin{array}{l}8.86 \\
9.00 \\
\end{array}$ \\
\hline d & $\mathbf{H}$ & $\mathrm{CH}_{2} \mathrm{CH}=\mathrm{CH}_{2}$ & - & 55 & 76 & $\mathrm{C}_{17} \mathrm{H}_{18} \mathrm{~N}_{2} \mathrm{O}_{4}$ & 314 & $\begin{array}{l}64.96 \\
65.00\end{array}$ & $\begin{array}{l}5.73 \\
6.00\end{array}$ & $\begin{array}{l}8.91 \\
9.00\end{array}$ \\
\hline e & H & $\mathrm{C}_{4} \mathrm{H}_{9}(\mathrm{n})$ & - & $75-6$ & 80 & $\mathrm{C}_{18} \mathrm{H}_{22} \mathrm{~N}_{2} \mathrm{O}_{4}$ & 330 & $\begin{array}{l}65.45 \\
65.40\end{array}$ & $\begin{array}{l}6.66 \\
6.50\end{array}$ & $\begin{array}{l}8.48 \\
8.50\end{array}$ \\
\hline $\mathrm{f}$ & $\mathrm{H}$ & $\mathrm{C}_{6} \mathrm{H}_{11}$ & - & $100-1$ & 75 & $\mathrm{C}_{20} \mathrm{H}_{24} \mathrm{~N}_{2} \mathrm{O}_{4}$ & 356 & $\begin{array}{l}67.41 \\
67.40 \\
\end{array}$ & $\begin{array}{l}6.74 \\
6.50 \\
\end{array}$ & $\begin{array}{l}7.88 \\
7.80 \\
\end{array}$ \\
\hline g & $\mathrm{H}$ & $\mathrm{C}_{6} \mathrm{H}_{5} \mathrm{CH}_{2}$ & - & 70 & 85 & $\mathrm{C}_{21} \mathrm{H}_{20} \mathrm{~N}_{2} \mathrm{O}_{4}$ & 364 & $\begin{array}{l}69.23 \\
69.00 \\
\end{array}$ & $\begin{array}{l}5.49 \\
5.50 \\
\end{array}$ & $\begin{array}{l}7.69 \\
8.00 \\
\end{array}$ \\
\hline $\mathrm{h}$ & $\mathrm{H}$ & $\mathrm{C}_{6} \mathrm{H}_{5} \mathrm{CO}$ & - & 65 & 92 & $\mathrm{C}_{21} \mathrm{H}_{18} \mathrm{~N}_{2} \mathrm{O}_{5}$ & 378 & $\begin{array}{l}66.66 \\
60.50 \\
\end{array}$ & $\begin{array}{l}4.76 \\
4.50 \\
\end{array}$ & $\begin{array}{l}7.40 \\
7.30 \\
\end{array}$ \\
\hline $\mathrm{i}$ & $\mathrm{H}$ & $\mathrm{C}_{6} \mathrm{H}_{5}$ & - & $154-5$ & 87 & $\mathrm{C}_{20} \mathrm{H}_{18} \mathrm{~N}_{2} \mathrm{O}_{4}$ & 350 & $\begin{array}{l}68.57 \\
68.60 \\
\end{array}$ & $\begin{array}{l}5.14 \\
5.00\end{array}$ & $\begin{array}{l}8.00 \\
8.00\end{array}$ \\
\hline
\end{tabular}


Table 1: Continued

\begin{tabular}{|c|c|c|c|c|c|c|c|c|c|c|}
\hline \multirow{2}{*}{$\begin{array}{c}\text { Comp. } \\
\text { No. }\end{array}$} & \multirow{2}{*}{$\mathrm{Z}$} & \multirow{2}{*}{$\mathbf{R}$} & \multirow{2}{*}{$R^{\prime}$} & \multirow{2}{*}{$\begin{array}{c}\text { M.P } \\
{ }^{\circ} \mathrm{C}\end{array}$} & \multirow{2}{*}{$\begin{array}{c}\text { Yield } \\
\%\end{array}$} & \multirow{2}{*}{$\begin{array}{l}\text { Molecular } \\
\text { formula }\end{array}$} & \multirow{2}{*}{ M.wt. } & \multicolumn{3}{|c|}{$\begin{array}{c}\text { Analysis } \\
\text { Calcd./found }\end{array}$} \\
\hline & & & & & & & & $\mathrm{C} \%$ & $\mathrm{H} \%$ & $\mathrm{~N} \%$ \\
\hline XI-a & $\mathrm{H}$ & $\mathrm{CH}_{3}$ & - & 70 & 65 & $\mathrm{C}_{17} \mathrm{H}_{22} \mathrm{~N}_{2} \mathrm{O}_{4}$ & 318 & $\begin{array}{l}64.15 \\
64.00 \\
\end{array}$ & $\begin{array}{l}6.91 \\
7.00 \\
\end{array}$ & $\begin{array}{l}8.80 \\
9.00 \\
\end{array}$ \\
\hline $\mathrm{b}$ & $\mathrm{H}$ & $\mathrm{C}_{2} \mathrm{H}_{5}$ & - & 45 & 62 & $\mathrm{C}_{18} \mathrm{H}_{24} \mathrm{~N}_{2} \mathrm{O}_{4}$ & 332 & $\begin{array}{l}65.06 \\
65.00\end{array}$ & $\begin{array}{l}7.22 \\
7.50\end{array}$ & $\begin{array}{l}8.43 \\
8.40\end{array}$ \\
\hline c & $\mathrm{H}$ & $\mathrm{C}_{3} \mathrm{H}_{7}(\mathrm{n})$ & $\cdots$ & 60 & 76 & $\mathrm{C}_{19} \mathrm{H}_{26} \mathrm{~N}_{2} \mathrm{O}_{4}$ & 346 & $\begin{array}{l}65.89 \\
66.00\end{array}$ & $\begin{array}{l}7.51 \\
7.60\end{array}$ & $\begin{array}{l}8.09 \\
8.00\end{array}$ \\
\hline d & $\mathbf{H}$ & $\mathrm{CH}_{2} \mathrm{CH}=\mathrm{CH}_{2}$ & - & 65 & 83 & $\mathrm{C}_{19} \mathrm{H}_{24} \mathrm{~N}_{2} \mathrm{O}_{4}$ & 344 & $\begin{array}{l}66.27 \\
66.50\end{array}$ & $\begin{array}{l}6.97 \\
7.00\end{array}$ & $\begin{array}{l}8.13 \\
8.00\end{array}$ \\
\hline e & $\mathrm{H}$ & $\mathrm{C}_{4} \mathrm{H}_{9}(\mathrm{n})$ & - & 65 & 60 & $\mathrm{C}_{20} \mathrm{H}_{28} \mathrm{~N}_{2} \mathrm{O}_{4}$ & 360 & $\begin{array}{l}66.66 \\
66.00 \\
\end{array}$ & $\begin{array}{l}7.77 \\
8.00 \\
\end{array}$ & $\begin{array}{l}7.77 \\
7.50 \\
\end{array}$ \\
\hline f & $\mathrm{H}^{\prime}$ & $\mathrm{C}_{6} \mathrm{H}_{11}$ &. & $120-1$ & 70 & $\mathrm{C}_{22} \mathrm{H}_{30} \mathrm{~N}_{2} \mathrm{O}_{4}$ & 386 & $\begin{array}{l}68.39 \\
68.30 \\
\end{array}$ & $\begin{array}{l}7.77 \\
5.50 \\
\end{array}$ & $\begin{array}{l}7.25 \\
7.30 \\
\end{array}$ \\
\hline g & $\mathrm{H}$ & $\mathrm{C}_{6} \mathrm{H}_{5} \mathrm{CH}_{2}$ & - & 65 & 75 & $\mathrm{C}_{23} \mathrm{H}_{26} \mathrm{~N}_{2} \mathrm{O}_{4}$ & 394 & $\begin{array}{l}70.05 \\
70.00\end{array}$ & $\begin{array}{l}6.59 \\
6.80 \\
\end{array}$ & $\begin{array}{l}7.10 \\
7.00\end{array}$ \\
\hline $\mathrm{h}$ & $\mathrm{H}$ & $\mathrm{C}_{6} \mathrm{H}_{5} \mathrm{CO}$ & - & 57 & 80 & $\mathrm{C}_{23} \mathrm{H}_{24} \mathrm{~N}_{2} \mathrm{O}_{4}$ & 408 & $\begin{array}{l}67.64 \\
67.50\end{array}$ & $\begin{array}{l}5.88 \\
6.00\end{array}$ & $\begin{array}{l}6.86 \\
7.00\end{array}$ \\
\hline $\mathrm{i}$ & H & $\mathrm{C}_{6} \mathrm{H}_{5}$ & - & 61 & 65 & $\mathrm{C}_{22} \mathrm{H}_{24} \mathrm{~N}_{2} \mathrm{O}_{4}$ & 380 & $\begin{array}{l}69.47 \\
69.50\end{array}$ & $\begin{array}{l}6.31 \\
6.20\end{array}$ & $\begin{array}{l}7.36 \\
7.20\end{array}$ \\
\hline
\end{tabular}

righting reflex method using phenobarbitone as a reference drug $^{24}$. Groups of six frogs were injected in the dorsal lymph sac with the test compounds or phenobarbitone in three graded doses. The animals were observed until loss of righting reflex and for further three hours later. The animal was considered asleep, if it showed loss of righting reflex during 60 minutes. The percentage of hypnotic response was calculated for each dose. The results of hypnotic activity are shown in Table (3).

\section{2- The anticonvulsant activity}

For evaluation of the anticonvulsant activity, the method of soaji-Echaqueod Lim was used $^{25}$. Frogs (Bufo-Regularis from Egypt) were used as experimental animals. Phenobarbitone sodium was used as a reference compound. Pentylenetetrazol was used as a convulsion inducer. The test compounds were suspended in water (water for injection) by the aid of few drops of Tween-80, phenobarbitone sodium and pentylenetetrazol were dissolved in water (water for injection) containing few drops of the same suspending agent. Frogs weighing 15-30 gm were randomly arranged in groups each 6 animals. Each of three graded doses of the test compounds as well as phenobarbitone were injected in the dorsal lymph sac of a group of animals. Forty-five minutes later,a convulsive dose of pentylenetetrazol $(320 \mathrm{mg} / \mathrm{kg})$ was injected in each animal. The animals were observed for further one hour for the occurrence of an episode of tonic convulsion. Animal not exhibited such convulsion during the observation time were considered protected, the results of anticonvulsant activity are shown in Table (4). 
Table 2: Spectral data of the new compounds (III, VII-XI).

\begin{tabular}{|c|c|}
\hline $\begin{array}{c}\text { Comp. } \\
\text { No. }\end{array}$ & $\begin{array}{c}\text { Spectral data } \\
\text { IR }\left(\mathrm{cm}^{-1}\right),{ }^{1} \operatorname{HNMR}(\delta, \mathrm{ppm}), \text { Mass }(\mathrm{m} / \mathrm{z}, \%)\end{array}$ \\
\hline III-a & $\begin{array}{l}\text { IR: } 1765,1745(\mathrm{CO} \text { of the esters at positions } 1 \text { and } 3), 1715(\mathrm{CO} \text { at position- }-4), 1675 \\
\text { (CO at position-2 of the quinazoinedione ring system). } \\
\text { 'HNMR: } 1.22\left(2 \mathrm{t}, 6 \mathrm{H}, 2-\mathrm{CH}_{2}-\mathrm{CH}_{3}\right), 1.5\left(\mathrm{~s}, 3 \mathrm{H},-\mathrm{CH}_{3}\right), 4.2\left(2 \mathrm{q}, 4 \mathrm{H}, 2-\mathrm{CH}_{2}-\mathrm{CH}_{3}\right) \\
\quad 4.75\left(\mathrm{~s}, 2 \mathrm{H}, \mathrm{N}-\mathrm{CH} 2-\mathrm{CO} \text { at position-1), } 4.90\left(\mathrm{~s}, 2 \mathrm{H}, \mathrm{N}-\mathrm{CH}_{2}-\mathrm{CO} \text { at position-3), }\right.\right. \\
\quad 7.30-8.50 \text { (m, } 4 \mathrm{H} \text {, aromatic protons). }\end{array}$ \\
\hline VII-b & $\begin{array}{l}\text { IR: } 1749(\mathrm{CO} \text { of the ester), } 1700(\mathrm{CO} \text { at position- } 4), 1660 \text { (CO at position- } 2 \text { of the } \\
\text { quinazolinedione ring system). } \\
{ }^{1} \mathrm{HNMR}: 1.39\left(\mathrm{t}, 3 \mathrm{H}, \mathrm{CH}_{2}-\mathrm{CH}_{3}\right), 3.91\left(\mathrm{q}, 2 \mathrm{H}, \mathrm{CH}_{2}-\mathrm{CH}_{3}\right), 4.98\left(\mathrm{~s}, 2 \mathrm{H}, \mathrm{N}-\mathrm{CH}_{2}-\mathrm{CO}\right) \text {, } \\
\quad 5.44\left(\mathrm{~s}, 2 \mathrm{H}, \mathrm{N}-\mathrm{CH}_{2}-\mathrm{C}_{6} \mathrm{H}_{5}\right), 7.30-8.50 \text { (m, } 8 \mathrm{H} \text {, aromatic protons). }\end{array}$ \\
\hline IX-a & $\begin{array}{l}\text { IR: } 1715\left(\mathrm{CO} \text { of the } \mathrm{OCO}-\mathrm{CH}_{3} \text { group), } 1700(\mathrm{CO} \text { at position- } 4), 1650(\mathrm{CO} \text { at }\right. \\
\text { position-4), } 1650 \text { (CO at position-2 of the quinazolinedione ring system). } \\
\text { MS: } 276\left(\mathrm{C}_{14} \mathrm{H}_{16} \mathrm{~N}_{2} \mathrm{O}_{4}, 12.52, \mathrm{M}^{+}\right), 252\left(\mathrm{C}_{12} \mathrm{H}_{16} \mathrm{~N}_{2} \mathrm{O}_{4}, 100, \text { Base peak), } 217\right. \\
\left(\mathrm{C}_{12} \mathrm{H}_{13} \mathrm{~N}_{2} \mathrm{O}_{2}, 50.44\right), 190\left(\mathrm{C}_{10} \mathrm{H}_{10} \mathrm{~N}_{2} \mathrm{O}_{2}, 59.44\right), 189\left(\mathrm{C}_{10} \mathrm{H}_{9} \mathrm{~N}_{2} \mathrm{O}_{2}, 30.80\right), 162 \\
\left(\mathrm{C}_{8} \mathrm{H}_{6} \mathrm{~N}_{2} \mathrm{O}_{2}, 40.00\right), 132\left(\mathrm{C}_{7} \mathrm{H}_{4} \mathrm{~N}_{2} \mathrm{O}, 15.1\right)\end{array}$ \\
\hline$X-b$ & $\begin{array}{l}\text { IR: } 2990-2875 \text { ( } \mathrm{CH} \text { stretching of the aliphatic side chain at position-3), } 1745 \text { ( } \mathrm{CO} \text { of } \\
\text { the } \\
\text { ester), } 1700 \text { (CO at position-4), } 1660(\mathrm{CO} \text { at position- } 2 \text { of the quinazolinedione } \\
\text { ring system. } \\
{ }^{1} \mathrm{HNMR} \text { : } 1.30\left(\mathrm{t}, 3 \mathrm{H}, \mathrm{CH}_{2}-\mathrm{CH}_{3}\right), 4.00-4.2\left(\mathrm{~m}, 1 \mathrm{H} \text { (allylic system), } \mathrm{CH}_{2}-\mathrm{CH}_{-}-\mathrm{CH}_{2}-\right), 4.8 \\
\quad\left(\mathrm{q}, \mathrm{CH}_{2}-\mathrm{CH}_{3}\right), 5.10-5.30\left(2 \mathrm{~d}, 4 \mathrm{H}, \mathrm{N}-\mathrm{CH}_{2}-\mathrm{CH}=\text { and }=\mathrm{CH}-\mathrm{CH}_{2}-\mathrm{CO}\right), 7.30-8.50 \\
\text { (m, } 9 \mathrm{H} \text {, aromatic protons). }\end{array}$ \\
\hline$X I-b$ & 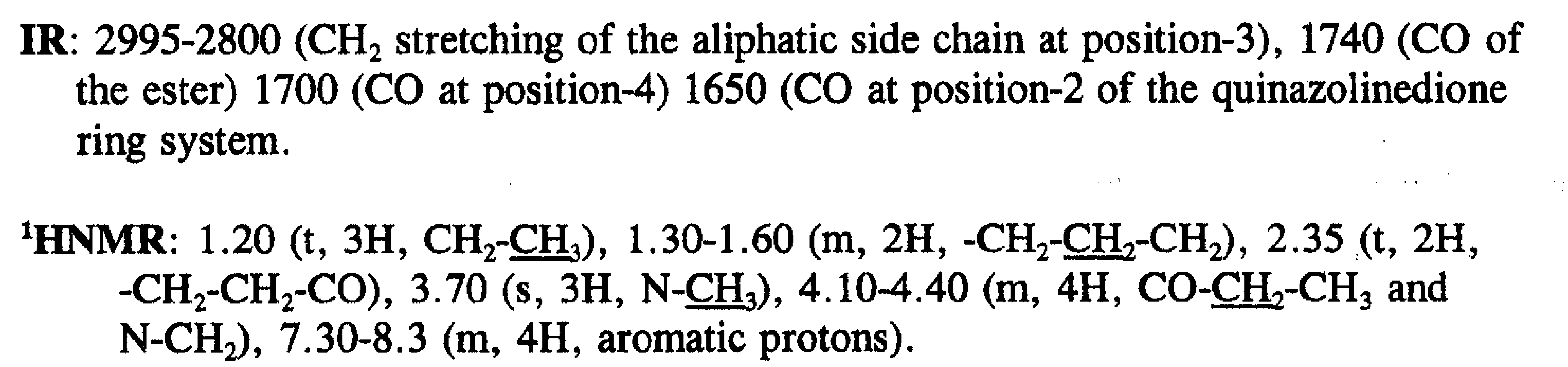 \\
\hline
\end{tabular}


Table 3: Hypnotic activity of $2,4(1 \mathrm{H}, 3 \mathrm{H})$ quinazolinedione derivatives.

\begin{tabular}{|c|c|c|c|c|c|c|c|c|c|c|c|}
\hline $\begin{array}{l}\text { Comp. } \\
\text { No. }\end{array}$ & $\begin{array}{c}\text { Dose } \\
\mathrm{mg} / \mathrm{kg}\end{array}$ & $\begin{array}{c}\text { Protection } \\
\%\end{array}$ & $\begin{array}{l}\text { Onset } \\
\text { Time } \\
\text { Min. } \\
\end{array}$ & $\mathrm{ED}_{50}$ & $\begin{array}{l}\text { Relative } \\
\text { Potency }\end{array}$ & $\begin{array}{l}\text { Comp. } \\
\text { No. }\end{array}$ & $\begin{array}{l}\text { Dose } \\
\mathrm{mg} / \mathrm{kg}\end{array}$ & $\begin{array}{c}\text { Protection } \\
\% \\
\end{array}$ & $\begin{array}{l}\text { Onset } \\
\text { Time } \\
\text { Min. } \\
\end{array}$ & $\mathrm{ED}_{50}$ & $\begin{array}{l}\text { Relative } \\
\text { Potency }\end{array}$ \\
\hline IIIa & $\begin{array}{r}50 \\
100 \\
150 \\
\end{array}$ & $\begin{array}{c}33.3 \\
66.6 \\
100 \\
\end{array}$ & 20 & 75 & 2.6 & XId & $\begin{array}{r}50 \\
100 \\
150 \\
\end{array}$ & $\begin{array}{c}33.3 \\
50 \\
100\end{array}$ & 30 & 94 & 2.1 \\
\hline b & $\begin{array}{r}50 \\
100 \\
150 \\
\end{array}$ & $\begin{array}{c}33.3 \\
66.6 \\
100 \\
\end{array}$ & 20 & 75 & 2.6 & e & $\begin{array}{l}100 \\
200 \\
300 \\
\end{array}$ & $\begin{array}{c}33.3 \\
66.6 \\
100 \\
\end{array}$ & 30 & 150 & 1.33 \\
\hline c & $\begin{array}{r}50 \\
100 \\
150 \\
\end{array}$ & $\begin{array}{r}33.3 \\
50 \\
100 \\
\end{array}$ & 20 & 43 & 4.6 & f & $\begin{array}{l}200 \\
300 \\
400 \\
\end{array}$ & $\begin{array}{r}33.3 \\
50 \\
100\end{array}$ & 45 & 273 & 0.72 \\
\hline d & $\begin{array}{r}25 \\
50 \\
100 \\
\end{array}$ & $\begin{array}{c}26.6 \\
50 \\
100 \\
\end{array}$ & 25 & 43 & 4.6 & g & $\begin{array}{l}100 \\
200 \\
300 \\
\end{array}$ & $\begin{array}{c}33.3 \\
66.6 \\
100 \\
\end{array}$ & 45 & 150 & 1.33 \\
\hline e & $\begin{array}{r}50 \\
100 \\
150 \\
\end{array}$ & $\begin{array}{c}33.3 \\
66.6 \\
100 \\
\end{array}$ & 30 & 75 & 2.6 & $\mathrm{~h}$ & $\begin{array}{l}200 \\
300 \\
400 \\
\end{array}$ & $\begin{array}{c}33.3 \\
66.6 \\
100 \\
\end{array}$ & 45 & 250 & 0.8 \\
\hline XIa & $\begin{array}{l}100 \\
200 \\
300 \\
\end{array}$ & $\begin{array}{c}33.3 \\
66.6 \\
100 \\
\end{array}$ & 40 & 150 & 1.33 & i & $\begin{array}{r}50 \\
100 \\
200 \\
\end{array}$ & $\begin{array}{c}33.3 \\
50 \\
100\end{array}$ & 25 & 94 & 2.1 \\
\hline b & $\begin{array}{l}100 \\
200 \\
300 \\
\end{array}$ & $\begin{array}{c}33.3 \\
66.6 \\
100 \\
\end{array}$ & 40 & 150 & 1.33 & $\mathrm{p}^{*}$ & $\begin{array}{l}150 \\
250 \\
300 \\
\end{array}$ & $\begin{array}{c}33.3 \\
66.6 \\
100 \\
\end{array}$ & 35 & 200 & 1.00 \\
\hline c & $\begin{array}{l}100 \\
200 \\
300\end{array}$ & $\begin{array}{c}33.3 \\
50 \\
100\end{array}$ & 30 & 150 & 1.33 & & & & & & \\
\hline
\end{tabular}

\footnotetext{
* Phenobarbitone.
} 
Table 4: Anticonvulsant activity of $2,4(1 \mathrm{H}, 3 \mathrm{H})$ quinazolinedione derivatives.

\begin{tabular}{|c|c|c|c|c|c|c|c|c|c|}
\hline $\begin{array}{c}\text { Comp. } \\
\text { No. }\end{array}$ & $\begin{array}{c}\begin{array}{c}\text { Dose } \\
\mathrm{mg} / \mathrm{kg}\end{array} \\
\end{array}$ & $\begin{array}{c}\text { Protection } \\
\% \\
\end{array}$ & $\mathrm{ED}_{50}$ & $\begin{array}{l}\text { Relative } \\
\text { Potency }\end{array}$ & $\begin{array}{c}\text { Comp. } \\
\text { No. } \\
\end{array}$ & $\begin{array}{c}\text { Dose } \\
\mathrm{mg} / \mathrm{kg}\end{array}$ & $\begin{array}{c}\text { Protection } \\
\% \\
\end{array}$ & $\mathrm{ED}_{s 0}$ & $\begin{array}{l}\text { Relative } \\
\text { Potency } \\
\end{array}$ \\
\hline IIIa & $\begin{array}{r}50 \\
100 \\
175 \\
\end{array}$ & $\begin{array}{c}50 \\
66.6 \\
100 \\
\end{array}$ & 50 & 1.8 & XId & $\begin{array}{l}150 \\
300 \\
400 \\
\end{array}$ & $\begin{array}{c}33.3 \\
66.6 \\
100 \\
\end{array}$ & 225 & 0.4 \\
\hline b & $\begin{array}{r}50 \\
100 \\
150 \\
\end{array}$ & $\begin{array}{c}16.6 \\
50 \\
100 \\
\end{array}$ & 100 & 0.9 & e & $\begin{array}{l}150 \\
300 \\
400 \\
\end{array}$ & $\begin{array}{c}33.3 \\
66.6 \\
100 \\
\end{array}$ & 225 & 0.4 \\
\hline c & $\begin{array}{r}50 \\
100 \\
150 \\
\end{array}$ & $\begin{array}{c}33.3 \\
66.6 \\
100 \\
\end{array}$ & 75 & 1.2 & g & $\begin{array}{l}200 \\
300 \\
400\end{array}$ & $\begin{array}{c}33.3 \\
50 \\
100 \\
\end{array}$ & 275 & 0.36 \\
\hline d & $\begin{array}{r}25 \\
50 \\
100 \\
\end{array}$ & $\begin{array}{c}33.3 \\
66.6 \\
100 \\
\end{array}$ & 40 & 2.2 & $\mathrm{i}$ & $\begin{array}{l}150 \\
250 \\
350 \\
\end{array}$ & $\begin{array}{c}33.3 \\
66.6 \\
100 \\
\end{array}$ & 200 & 0.5 \\
\hline e & $\begin{array}{r}50 \\
100 \\
150 \\
\end{array}$ & $\begin{array}{c}33.3 \\
66.6 \\
100 \\
\end{array}$ & 75 & 1.2 & $\mathrm{p}^{*}$ & $\begin{array}{r}75 \\
125 \\
200 \\
\end{array}$ & $\begin{array}{c}33.3 \\
66.6 \\
100 \\
\end{array}$ & 100 & 1.0 \\
\hline XIa & $\begin{array}{l}200 \\
300 \\
400 \\
\end{array}$ & $\begin{array}{c}33.3 \\
50 \\
100 \\
\end{array}$ & 27.5 & 0.36 & & & & & \\
\hline b & $\begin{array}{l}150 \\
250 \\
350 \\
\end{array}$ & $\begin{array}{c}33.3 \\
66.6 \\
100 \\
\end{array}$ & 200 & 0.50 & & & & & \\
\hline c & $\begin{array}{l}150 \\
300 \\
400\end{array}$ & $\begin{array}{c}16.6 \\
50 \\
100\end{array}$ & 275 & 0.36 & & & & & \\
\hline
\end{tabular}

* Phenobarbitone.

\section{Structure activity relationship}

From the results of the pharmacological testing (Tables 3 and 4), it has been shown that the introduction of an electron repelling methyl group at the 6-position of the $2,4-(1 \mathrm{H}, 3 \mathrm{H})$ quinazolinedione ring system has no influence on the pharmacological activity. On the other hand, substitution of this $\mathrm{CH} 3$ group at the 6-position by an electron withdrawing bromide atom practically inhibited the pharmacological activity of the original compounds. Furthermore, insertion of a propionate, crotonate, or hexonate esters at the 3-position through elongation of the side chain was found to decrease the anticonvulsant and hypnotic activities relative to our previous results concerning the Ethyl 1-ethyland 1-benzyl-2,4-(1H, 3H) quinazolinedion-3-yl acetate $^{5,7}$, which has been proved to be the most potent anticonvulsant and hypnotic compounds out of these series. 


\section{REFERENCES}

1- S.Johne, Proq. Drug. Res., 26, 260 (1982).

2- S.Hayao, H.J.Havera, W.G.Strycker, T.J.Leipzi, r.A.Klup and H.E.Hartzler, J. Med. Chem., 8, 807 (1965).

3- B.Bas and R.Mukherjee, J. Indian Chem. Soc., 40, 35 (1963).

4- D.G.Wenzel, J. Am. Pharm. Assoc. Sci., Ed. 44, 550 (1955).

5- A.R.E.N.Ossman, A.G.N.Ossman, and A.G.A. El-Helby, Bull. Pharm. Sci., Assiut Univ., 9 (1), 105 (1986).

6- M.A.E. Aziza, F.M.Salama, M.A.Amin and A.G.A.El-Helby, Az. J. Pharm. Sci., 9, 63-74 (1992).

7- A.G.A.El-Helby, Egypt. J. Pharm. Sci, (Part 1), (1995) in Press.

8- A.E.Agronov and U.C.Shabarov, Practical Organic Chemistry, publishing house Chemistry, Moscow, 78 (1974).

9- J.Houben and W.Brassert, Ber., 39, 3233 (1906).

10- H.Fujimura and Y.Yama Kaw (Takeda Chemical Industries LTD), Japan 11, 454 (66), (Ch. 16. C 86) June 25, Appl. June 3 (1994), C.A., 65, 1528/d (1966).

11- D.H.Hey and D.G.Turpin, J. Chem. Soc., 19, 2471-81 (1954).

12- F.Ullmann, Ber., 36, 2383 (1903).

13- P.G.Pastor, C.Blanchard, C.Montginocl, E.Torreillles, L.Giral and A.Takier, Bull. Soc. Chem. France, 5-6 (pt. 2) 331-8 (1976).

14- S.Somasekhara, V.S.Dighe, S.L. Mukherjee, Curr. Sci., 31-7(18), 529-30 (Eng) (1968).

15- N.A.Lang and F.E.Sheibley, Organic Synthesis Coll., Vol. II, Johne Wiley and Sons. Inc. New York, N.Y., p. 79 (1947).
16- M.Susse and S.Johne, Monatsh Chem., 118,71 (1987).

17- N.A.Lang and F.E.Sheibley, J. Am. Chem. Soc., 55, 2113 (1933).

18- M.Susse and S.Johne, Monatsh Chem., 117, 499 (1986).

19- M.Saita and M.Tasiji, Nippon Yakarigokzasshi,76 (8), 675 (1980), C.A. 94, 76684 n (1980).

20- M.Kalifa, A.G.N.Osman, M.G.Ibrahim, A.R.E.N.Ossman and M.A.Ismail, Pharmazie, 37 (1982).

21- N.Kanji, N.Akira, M.Toshiharu, I.Hiroyuki, F.Hajime, (Hisamitu pharmaceutical Co. Inc). Japan Kohai, 74, 110, 683 (cl. 16E464), 22 Oct. 1974. Appl. 73, 28, 341 O. \& Mar 1973, through C.A., Vol. 82, 171021e (1975).

22- J.Vandenberk, L.Kennis, A.M.Van der, A.Van Heertum; (Janssen pharmaceutical, N.V) U.S 4, 335, 127 (Cl. 424-251, Co 7D 401/06) 15 Jun. 1982, US Apple., 1, 493, 08 Jan. 1979, 21 pp. Cont. in Part of U.S, Ser. No. 1, 493 abandoned. C.A., 97, 216203u (1982).

23- J.Vandenberk, L.E.J.Kennis, M.J.M.C.Van Der Aa, A.H.M.T.Van Heertum, (Jensen pharmaceutical N.V.) Eur. Pat. Appl. 13, 612 (Cl. Co 7 D401/06). 23 Jul 1980, US Appl. 1, 493, 08 Jan. 1979, C.A. 94, 65718a (1981).

24- R.A.Turner, "Screening Methods in Pharmacology" Academic Press, London, New York pp. (a) 69, (b) 164 (1955).

25- E.Soaje-Echaque and R.K.S.Lim, J. Pharmacol. Exper. Ther., 138, 224 (1962). 